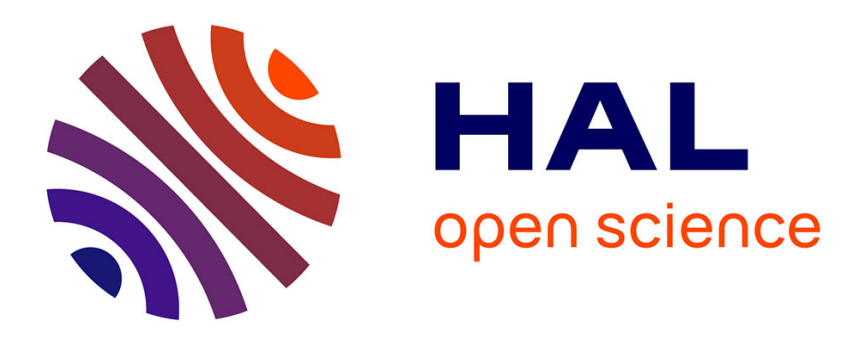

\title{
Explorer et gérer les espaces de discussion sur le travail
}

Damien Collard

\section{To cite this version:}

Damien Collard. Explorer et gérer les espaces de discussion sur le travail. Gérer et Comprendre. Annales des Mines, 2019, 4 (138), pp.21-30. 10.3917/geco1.138.0021 . hal-02453853

\section{HAL Id: hal-02453853 \\ https://u-bourgogne.hal.science/hal-02453853}

Submitted on 3 Dec 2021

HAL is a multi-disciplinary open access archive for the deposit and dissemination of scientific research documents, whether they are published or not. The documents may come from teaching and research institutions in France or abroad, or from public or private research centers.
L'archive ouverte pluridisciplinaire HAL, est destinée au dépôt et à la diffusion de documents scientifiques de niveau recherche, publiés ou non, émanant des établissements d'enseignement et de recherche français ou étrangers, des laboratoires publics ou privés. 


\title{
Explorer et gérer les espaces de discussion sur le travail
}

\author{
Par Damien COLLARD \\ Université de Franche-Comté / Centre de recherche en gestion des organisations de \\ l'Université de Bourgogne
}

Dans cet article, nous nous intéressons aux espaces de discussion consacrés au travail, à leurs caractéristiques, mais aussi à ce qui se joue et à ce qui se dit dans ces lieux. Une recherche-action menée dans un service de gériatrie nous a conduits à explorer les dynamiques communicationnelles qui s'instaurent dans ces espaces et à réfléchir à leur ingénierie, ce qui induit plusieurs questions. Tout d'abord, comment les définir et les repérer quand on sait qu'ils sont potentiellement très nombreux ? Ensuite, faut-il les structurer, les équiper et les connecter entre eux ? Enfin, faut-il proposer la mise en place de nouveaux espaces de discussion, et dans quel but?

\section{Introduction}

La réflexion sur les espaces de discussion consacrés au travail s'inscrit dans une filiation ancienne, comme le soulignent Abord de Chatillon et Desmarais (2016) qui rappellent que l'expression des travailleurs était, à l'origine, une revendication portée par le mouvement de mai 1968. Cette revendication trouvera un écho favorable dans les lois Auroux de 1982, qui instituent un droit à l'expression directe et collective des salariés sur le contenu et l'organisation de leur travail, et qui donneront naissance aux groupes d'expression (Borzeix, 1987). S'ensuivront les cercles de qualité quelques années plus tard, puis, à partir des années 1990, la prolifération de « dispositifs d'incitation à la parole des salariés » (Borzeix, 2001, p. 80) dont les visées sont multiples : générer des innovations, assurer une meilleure coordination, analyser les pratiques professionnelles ${ }^{1}$, etc.

Plus récemment, a émergé l'idée que certains dispositifs - qualifiés d'« espaces de discussion »- permettraient de mieux réguler les tensions qui naissent du travail et de préserver la santé des salariés, de par les dynamiques communicationnelles qui s'instaurent dans ces lieux. Plusieurs travaux récents en sciences de gestion tendent d'ailleurs à confirmer cette hypothèse (Richard, 2012 ; Abord de Chatillon et Desmarais, 2017). Si, à l'origine, cette idée a été promue principalement par la psychodynamique du travail (Dejours, 1992, 1998, 2013), puis par la clinique de l'activité (Clot, 2008, 2010), elle irrigue aujourd'hui les travaux en sciences de gestion qui portent sur l'ingénierie des espaces de discussion (Detchessahar, 2009, 2011a, 2011b, 2013 ; Detchessahar et al., 2009, 2015, 2017 ; Briest-Breda et al., 2017 ; Bellini et al., 2018). Par ailleurs, elle se traduit par le déploiement, dans les organisations, de nouvelles pratiques d'intervention et de nouveaux outils, sous l'égide de l'Agence nationale d'amélioration des conditions de travail (ANACT) notamment, qui s'est emparée du thème des espaces de discussion (Conjard et Journoux, 2013 ; Conjard, 2015).

\footnotetext{
${ }^{1}$ Voir à ce sujet le numéro 11 de la Nouvelle Revue de Psychosociologie consacré aux « groupes d'analyse des pratiques » (un type de dispositif qui a suscité un véritable engouement, dans le secteur médico-social notamment).
} 
II ressort de la littérature sur l'ingénierie des espaces de discussion, quand on la confronte aux approches cliniques du travail, un certain nombre de questions. Tout d'abord, comment définir ces espaces et les repérer quand on sait qu'ils sont potentiellement très nombreux ? Ensuite, faut-il les structurer, les équiper et les connecter entre eux ? Enfin, faut-il proposer la mise en place de nouveaux espaces de discussion sur le travail, et dans quel but ? C'est à ces questions qu'entend s'atteler cet article, à partir des premiers résultats d'une recherche-action menée au sein d'un service de gériatrie. Après avoir exposé notre cadre théorique, nous présenterons notre terrain et notre méthodologie, puis nos résultats empiriques et nos propositions, avant de les discuter.

\section{La littérature sur les espaces de discussion comme cadre conceptuel}

La psychodynamique du travail a particulièrement bien étudié les rapports entre la délibération sur le travail et la préservation de la santé (Dejours, 1992, 1998, 2013). Pour Dejours (1992), cette délibération se produit dans un lieu dénommé « espace de discussion » ou « espace de délibération », dans lequel se déploie une « activité déontique ", c'est-à-dire de construction de règles de travail. Cette activité est, selon lui, un facteur de préservation de la santé mentale des travailleurs. Si une part de la délibération sur le travail prend place dans des réunions formelles, la majeure partie se déroule dans des lieux informels qui sont avant tout voués à la convivialité (Dejours, 1992, 2013). C'est en effet dans les interstices que les travailleurs peuvent partager émotions et affects. C'est ce qu'a constaté Molinier $(2008,2013)$ dans ses travaux sur les aides-soignantes et les infirmières. Elle a notamment observé que les soignantes n'échangeaient pas que des informations techniques lors des discussions informelles, mais également leurs doutes, leurs inquiétudes, leurs affects (dégoût, répulsion, attirance, impuissance, etc.). Par conséquent, il convient d'être attentif à ce qui se passe dans les interstices, « ces lieux où s'entremêlent des échanges concernant les pratiques professionnelles mais aussi la vie privée. (...) L'apparente banalité des paroles qui s'y échangent recouvre en vérité des affects puissants ; c'est, en effet, dans les interstices, que les membres d'une institution expérimentent le plaisir de vivre ensemble » (Fustier, 2012, p. 95). L'analyse du travail doit donc braquer le projecteur sur ces interstices.

Le point de vue défendu par Mathieu Detchessahar est, lui, très différent : d'une part parce qu'il ne s'intéresse pas aux espaces informels ; d'autre part parce que, selon lui, « l'intervenant est moins là pour proposer des méthodes d'analyse du travail que pour mettre en place et médier de nouveaux espaces » (Detchessahar et al., 2015, p. 79), en commençant par l'institutionnalisation d'espaces de discussion de niveau stratégique, avant de se tourner vers le travail opérationnel. Pour Detchessahar, la discussion sur le travail nécessite en effet une ingénierie de la discussion capable de prendre en charge les tensions qui naissent de l'activité réelle et de soutenir un processus d'enquête ${ }^{2}$ (Detchessahar et al., 2017). Ces espaces ont plusieurs caractéristiques, selon Detchessahar et al. (2015) : ils sont centrés sur l'activité

\footnotetext{
${ }^{2}$ Selon Journé (2007, p. 3), « L'enquête est provoquée par le doute attaché au caractère indéterminé de la situation. Les situations "indéterminées" sont dites "perturbées, ambigües, confuses, pleines de tendances contradictoires, obscures, etc." (Dewey, 1993, p. 170) L'enquête ouverte par le doute s'inscrit dans un projet d'action ; il porte donc autant sur les éléments constitutifs de la situation que sur les difficultés à entrevoir ses évolutions potentielles... " Le processus d'enquête est donc étroitement associé au concept de situation (Journe et Raulet-Croset, 2008).
} 
concrète ; ils sont pilotés et animés par un responsable hiérarchique ; ils s'inscrivent dans le long terme ; ils sont outillés. Toute réunion ne s'apparente donc pas à un espace de discussion. Cela suppose en effet une ingénierie « qui définisse clairement les participants, la fréquence, les modalités d'animation managériale et les connexions avec le reste de l'organisation » (Detchessahar et al., 2017, p. 36). Ce faisant, cette ingénierie explore avant tout les dimensions cognitive et politique de la délibération sur le travail, mais pas la dimension affective et émotionnelle, comme le notent Briest-Breda et al. (2017) qui proposent de renouveler l'ingénierie des espaces de discussion en intégrant cette dimension.

\section{Présentation du terrain et de la méthodologie}

Notre terrain est un service spécialisé en soins de suite et de réadaptation gériatriques rattaché à un hôpital public. II se compose de deux unités fonctionnelles de 36 lits chacune. Chaque unité est pilotée par un binôme constitué d'un médecin et d'un cadre de santé. Le premier organise l'activité médicale et coordonne le travail des médecins. Le second anime une équipe composée d'infirmiers et d'aidessoignants. Nous avons répondu à une demande d'intervention émanant de l'un de ces binômes qui souhaitait améliorer la prise en charge des patients accueillis dans l'unité et renforcer la coordination ainsi que la communication au niveau de l'équipe. II était notamment attendu de l'intervenant qu'il s'appuie sur les suggestions de l'équipe pour formuler des propositions concrètes en matière d'organisation du travail. Pour répondre à cette demande, nous avons déployé une recherche-action qui s'est déroulée en deux temps : (1) une phase d'enquête sur le terrain, qui a donné lieu à la remise d'un rapport au comité de suivi de la recherche ${ }^{3}$; (2) une phase de discussion des résultats de l'enquête.

Durant la première phase de la recherche, nous avons mené 25 entretiens semidirectifs auprès des soignants et réalisé une analyse de l'activité des aides-soignants et des infirmiers. Pour ce faire, nous nous sommes inspirés de l'ergonomie et avons réalisé des suivis d'activités (Guerin et al., 1997) pour analyser les pratiques langagières orales des soignants en les suivant dans des «cours d'action " ${ }^{4}$, comme l'ont fait avant nous Grosjean et Lacoste (1999) dans leur étude du travail des infirmières. En l'espace de trois mois, nous avons ainsi pu suivre les aides-soignants et les infirmiers dans leur activité pendant une cinquantaine d'heures environ. Nous avons de ce fait observé des centaines d'interactions sociales et avons pu en retranscrire 82 (intégralement ou partiellement) dans notre journal de bord. Certaines sont extrêmement brèves (elles durent quelques secondes), tandis que d'autres sont très longues (plusieurs dizaines de minutes pour certaines d'entre elles). Deux types d'interactions ont été retranscrits : d'une part, 39 interactions soignants-patients (dont nous ne parlerons pas dans cet article), et, d'autre part, 43 interactions entre soignants (impliquant au moins deux soignants, mais pouvant mobiliser jusqu'à une dizaine de professionnels), interactions qui constituent notre matériau de base pour repérer et catégoriser les espaces de discussion.

Pour réaliser cet inventaire des espaces de discussion, nous nous sommes appuyés sur une définition ad hoc de l'espace de discussion. Nous considérons qu'il s'agit

\footnotetext{
${ }^{3}$ Cette instance se compose du médecin responsable de l'équipe médicale, de la cadre de santé, d'une infirmière, d'un aide-soignant et du chercheur.

${ }^{4}$ Le « cours d'action » est « l'activité d'un acteur déterminé (...) qui est significative pour ce dernier, c'est-à-dire montrable, racontable et commentable par lui à tout instant de son déroulement à un observateur-interlocuteur » (Theureau et Jeffroy, 1994, p. 19).
} 
d'un espace (formel ou informel) dans lequel sont impliqués au moins deux soignants, qui donne lieu à une discussion en lien avec l'activité, et qui permet de réguler les tensions et les contradictions qui naissent de cette activité. Cette définition a été forgée en amont de notre enquête de terrain en confrontant trois cadres théoriques : les travaux sur l'ingénierie des espaces de discussion, la psychodynamique du travail et la clinique de l'activité. Elle a l'avantage de relier entre eux les trois invariants repérés par Abord de Chatillon et Desmarais (2016) dans leur revue de littérature consacrée aux liens entre les espaces de discussion et la santé au travail. Selon eux, ces espaces « sont centrés sur le travail ; ils permettent un échange entre acteurs ; ils permettent de réguler l'activité » (Abord de Chatillon et Desmarais, 2016, p. 9). Pour les repérer, nous sommes partis des 43 interactions entre soignants consignées dans notre journal de bord, parmi lesquelles nous avons pu identifier celles qui pouvaient s'apparenter à des espaces de discussion au sens de notre définition. II s'agit de celles qui répondent effectivement au moins aux trois premiers critères présentés dans le tableau 1 ci-dessous ; quant aux trois autres critères mentionnés dans ce tableau, ils nous ont permis de distinguer différents types d'espaces, selon qu'il s'agisse d'espaces structurés ou non (cf. critère $n^{\circ} 4$ ), pilotés ou non (cf. critère $n^{\circ} 5$ ), qui émergent spontanément ou du fait de la présence du chercheur (cf. critère $n^{\circ} 6$ ). Ces critères nous ont donc permis de déterminer et de caractériser différents espaces de discussion.

\begin{tabular}{|l|l|}
\hline Critère $\mathbf{n}^{\circ 1}$ & $\begin{array}{l}\text { Situation indéterminée : la situation évoquée et/ou traitée par les soignants est floue, } \\
\text { incertaine, confuse et incite les soignants à s'engager collectivement dans un processus } \\
\text { d'enquête. }\end{array}$ \\
\hline Critère $\mathbf{n}^{\circ 2}$ & $\begin{array}{l}\text { Activité complexe : l'activité communicationnelle dans laquelle les soignants s'engagent } \\
\text { est complexe, d'une part parce que la situation évoquée et/ou traitée est indéterminée, } \\
\text { d'autre part parce que le processus d'enquête dans lequel ils s'impliquent est lui-même } \\
\text { complexe. }\end{array}$ \\
\hline Critère $\mathbf{n}^{\circ} \mathbf{3}$ & $\begin{array}{l}\text { Forte charge émotionnelle : la situation évoquée et/ou traitée induit chez au moins un } \\
\text { soignant des émotions et/ou des affects négatifs (ex : peur, angoisse, colère, dégoût, } \\
\text { répulsion, tristesse, etc.) qu'il partage avec ses collègues. }\end{array}$ \\
\hline Critère $\mathbf{n}^{\circ} \mathbf{4}$ & $\begin{array}{l}\text { Activité structurée : l'activité communicationnelle dans laquelle les soignants } \\
\text { s'impliquent s'inscrit dans un cadre formel et est outillée (grâce à des supports écrits } \\
\text { notamment, papiers ou informatiques...). }\end{array}$ \\
\hline Critère $\mathbf{n}^{\circ} \mathbf{5}$ & $\begin{array}{l}\text { Activité pilotée par le management : le cadre de santé ou un médecin anime et structure } \\
\text { la discussion. }\end{array}$ \\
\hline Critère $\mathbf{n}^{\circ} \mathbf{6}$ & $\begin{array}{l}\text { Activité induite par la présence du chercheur : la présence et/ou les questions du } \\
\text { chercheur incite(nt) les soignants à s'engager dans un échange. }\end{array}$ \\
\hline
\end{tabular}

Tableau 1 : Critères permettant de déterminer et de caractériser les espaces de discussion

Les six critères présentés dans ce tableau sont issus de la littérature : les deux premiers découlent des réflexions de Journé (2007) et de Journe et Raulet-Croset (2008) sur le processus d'enquête ; le troisième critère est issu des recherches de Molinier $(2008,2013)$ sur les soignantes en gériatrie ; les deux critères suivants découlent des travaux sur l'ingénierie des espaces de discussion ; le dernier critère est inspiré des travaux de Clot (2008). Si les trois premiers critères nous ont permis d'identifier, parmi les interactions recueillies, celles qui correspondent à des espaces de discussion, les trois derniers nous ont permis d'affiner l'analyse en caractérisant 
cinq types d'espaces que nous présentons de manière succincte dans le tableau 2 ci-dessous.

\begin{tabular}{|c|c|}
\hline ED 1 & $\begin{array}{l}\text { Interactions « naturelles » qui se déroulent dans les interstices (couloirs, salle de pause, hors des } \\
\text { murs de l'hôpital lors des pauses cigarettes, etc.). Elles donnent lieu à un processus d'enquête (cf. } \\
\text { critère } n^{\circ} 1 \text { ), et donc à une forme de retour réflexif sur les tensions et les contradictions qui naissent } \\
\text { de l'activité - c'est-à-dire que les soignants évoquent les difficultés rencontrées dans le travail (de } \\
\text { manière explicite, implicite ou allusive) et croisent leur point de vue (cf. critère } n^{\circ} 2 \text { ) ; font part de } \\
\text { leur ressenti, ouvertement ou à demi-mots (cf. critère } n^{\circ} 3 \text { ). }\end{array}$ \\
\hline ED 2 & $\begin{array}{l}\text { Interactions «provoquées » par la présence du chercheur (cf. critère } n^{\circ} 6 \text { ) et se déroulant dans les } \\
\text { interstices. Elles donnent lieu à un processus d'enquête (cf. critère } n^{\circ} 1 \text { ) et se traduisent par une } \\
\left.\text { activité communicationnelle complexe (cf. critère } n^{\circ} 2 \text { ) et par un partage émotionnel (cf. critère } n^{\circ} 3\right) \text {. }\end{array}$ \\
\hline ED 3 & $\begin{array}{l}\text { Transmissions orales (réalisées dans le cadre des relèves inter-équipes) qui donnent lieu à un } \\
\text { processus d'enquête (cf. critère } n^{\circ} 1 \text { ) et à un partage émotionnel (cf. critère } n^{\circ} 3 \text { ). L'activité } \\
\text { communicationnelle qui se déploie lors de ces transmissions est complexe (cf. critère } n^{\circ} 2 \text { ) et est } \\
\text { structurée (cf. critère } n^{\circ} 4 \text { ). }\end{array}$ \\
\hline ED 4 & $\begin{array}{l}\left.\text { Staffs (animés par un médecin) qui donnent lieu à une discussion approfondie (cf. critère } n^{\circ} 2\right) \text { et à } \\
\left.\text { un processus d'enquête (cf. critère } n^{\circ} 1\right) \text {. L'activité communicationnelle qui se déroule dans ces } \\
\left.\left.\text { staffs est structurée (cf. critère } n^{\circ} 4\right) \text { et est pilotée (cf. critère } n^{\circ} 5\right) \text {. Elle permet aux soignants de } \\
\text { partager leur ressenti (cf. critère } 3 \text { ). }\end{array}$ \\
\hline ED 5 & $\begin{array}{l}\text { Echanges approfondis entre soignants (cf. critère } n^{\circ} 2 \text { ) lors des réunions organisées par } \\
\text { l'encadrement intermédiaire, qui portent sur l'activité et l'organisation du travail, et qui génèrent un } \\
\text { processus d'enquête (cf. critère } n^{\circ} 1 \text { ). L'activité communicationnelle qui se déploie lors de ces } \\
\text { réunions est structurée (cf. critère } n^{\circ} 4 \text { ) et est pilotée (cf. critère } n^{\circ} 5 \text { ). Lors de ces échanges, les } \\
\text { soignants n'hésitent pas évoquer leur ressenti (cf. critère } n^{\circ} 3 \text { ). }\end{array}$ \\
\hline
\end{tabular}

Tableau 2 : Les espaces de discussion (ED) en gériatrie

On voit que ces espaces de discussion peuvent être structurés (trois cas) ou non, pilotés par le management (deux cas) ou non, et qu'ils sont rarement « provoqués » par la présence du chercheur (un seul cas). Le tableau 3 ci-dessous reprend chaque cas au regard des six critères énoncés.

\begin{tabular}{|l|c|c|c|l|l|l|}
\hline & $\begin{array}{l}\text { Situation } \\
\text { indéterminée }\end{array}$ & $\begin{array}{l}\text { Activité } \\
\text { complexe }\end{array}$ & $\begin{array}{l}\text { Forte charge } \\
\text { émotionnelle }\end{array}$ & $\begin{array}{l}\text { Activité } \\
\text { structurée }\end{array}$ & $\begin{array}{l}\text { Activité pilotée } \\
\text { par le } \\
\text { management }\end{array}$ & $\begin{array}{l}\text { Activité induite } \\
\text { par la présence du } \\
\text { chercheur }\end{array}$ \\
\hline ED 1 & $\checkmark$ & $\checkmark$ & $\checkmark$ & & & \\
\hline ED 2 & $\checkmark$ & $\checkmark$ & $\checkmark$ & & & $\checkmark$ \\
\hline ED 3 & $\checkmark$ & $\checkmark$ & $\checkmark$ & $\checkmark$ & & \\
\hline ED 4 & $\checkmark$ & $\checkmark$ & $\checkmark$ & $\checkmark$ & $\checkmark$ & \\
\hline ED 5 & $\checkmark$ & $\checkmark$ & $\checkmark$ & $\checkmark$ & $\checkmark$ & \\
\hline
\end{tabular}

Tableau 3 : Synthèse

\section{Résultats empiriques et préconisations}

Dans le but de donner à voir au lecteur ce qui se joue et ce qui se dit dans ces espaces, nous allons présenter deux conversations issues de notre enquête. L'une prend place dans un cadre formel, tandis que l'autre est informelle. La première conversation se déroule lors des transmissions orales entre aides-soignants (cf. ED 3 dans le tableau 2) et a lieu dans la salle de pause des personnels paramédicaux. On y voit quatre aides-soignantes échanger des informations sur la situation des patients, mais aussi évoquer leurs conditions de travail. Le début et la fin de cette conversation sont retranscrits dans l'encadré ci-dessous. 


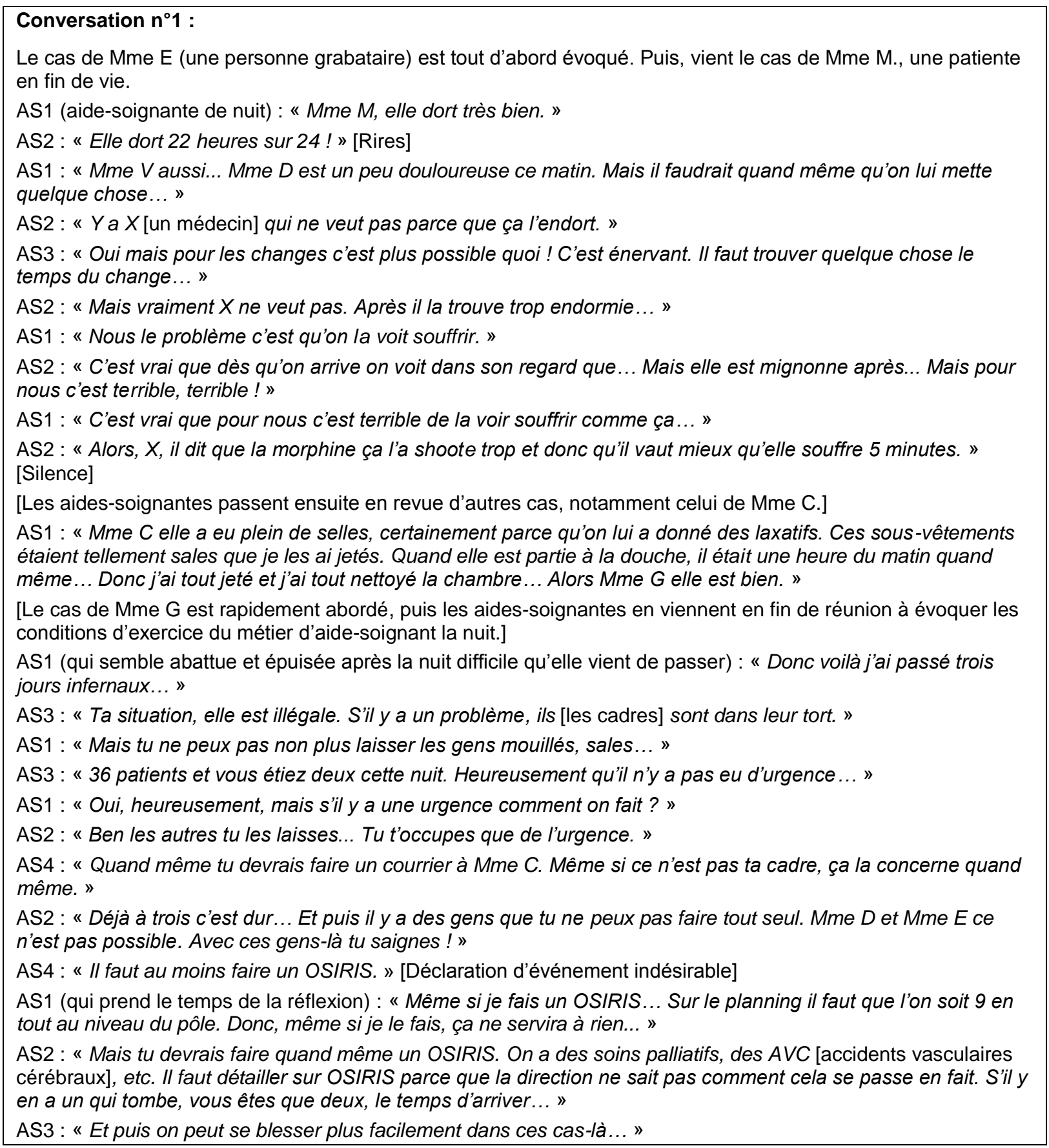

La tonalité de cette conversation est grave, malgré une touche d'humour apportée par AS2 en tout début de réunion. Grave parce que les cas évoqués constituent de véritables drames humains du fait des souffrances (physiques et/ou morales) endurées par les personnes âgées et de leur état de santé (personnes grabataires, en fin de vie, etc.), mais aussi du fait des conditions de travail des aides-soignantes, qui sont confrontées au quotidien à la maladie, à la mort, à des corps qui dysfonctionnent, aux déjections et à diverses souillures... Ces situations font naître une souffrance éthique chez les aides-soignantes, soit parce que les soins d'hygiène et de confort qu'elles dispensent induisent de la souffrance chez la personne âgée (le cas de Mme D est à cet égard emblématique), soit parce qu'elles n'arrivent pas à répondre à toutes les sollicitations émanant de leur environnement. 
La deuxième conversation (cf. ED 1 dans le tableau 2) se déroule dans un couloir et implique trois aides-soignants. II y est notamment question de Monsieur M qui, la veille, a été particulièrement agressif avec l'équipe de nuit, une infirmière ayant notamment reçu un coup au visage. La situation n'est une surprise pour personne, Monsieur M étant connu pour son agressivité. Nous apprenons, à cette occasion, que le cadre de nuit a déclaré l'incident comme « événement indésirable » dans l'outil informatique prévu à cet effet, d'autant plus qu'aucun des soignants n'a pensé à remettre la contention de Monsieur $\mathrm{M}$. après la toilette du matin, alors même qu'il s'agit d'une prescription médicale.

\footnotetext{
Conversation $\mathrm{n}^{\circ} \mathbf{2}$ :

AS1 : «Le problème, à l'origine, c'est que AS4 n'est pas allée chercher la contention. »

AS2 : « (...) Et entre la journée et la nuit personne n'a eu le réflexe de mettre la contention ?»

AS1 : « II n'y a pas que ça. Il a été méchant. Il a tapé sur une infirmière. Tout ça figure dans l'ordinateur. (...). Mais le problème c'est qu'il y a une prescription médicale pour une contention. Madame L [la cadre supérieure du pôle gériatrie] l'a bien dit. On est tous en tort ! » (...)

AS3 (qui était le binôme d'AS4 le jour de l'incident) : « Mais moi j'ai percuté car j'ai dit à AS4 qu'il avait une contention. Et elle m'a dit qu'elle allait la chercher. Après elle n'est pas allée la chercher... Après moi, honnêtement, je ne suis pas revenue parce qu'elle m'a dit qu'elle allait le faire... "

AS2 : « La contention c'est le truc qu'il faut pas zapper... »

AS1 : " Mais il y a des soignants qui ne la mettent pas ! C'est pareil pour Monsieur F. Il a chuté plusieurs fois, dont la semaine dernière. Le problème, ce sont les soignants qui ne veulent pas la mettre. Mais moi je la mets qu'il soit content ou pas. "

AS2 : "Mais ce n'est pas "contention si besoin" pour lui ? »

AS1 : «Non c'est $Y$ [une infirmière] qui ne veut pas que l'on mette la contention pour lui. Mais comme il a chuté plusieurs fois le médecin a à nouveau prescrit la contention. Après, comme je disais à la cadre, il y a le problème que les contentions sont au deuxième étage... Alors s'il faut courir à chaque fois pour aller chercher la clé... que je n'ai même pas trouvée d'ailleurs. (...) "

AS2 : «Après il y a une référente contention... que l'on voit de temps en temps. Et le problème c'est que les contentions elles ne sont pas triées... »

La conversation se poursuit sur les problèmes organisationnels liés à la gestion des contentions.
}

Ces deux conversations donnent à voir des professionnels qui s'investissent pleinement dans un processus d'enquête en vue de résoudre les problèmes qui se posent dans le travail. Mais dans la mesure où ceux-ci ne trouvent pas de solutions et que le processus d'enquête déborde le cadre de ces deux conversations ${ }^{5}$, il convient de réfléchir non seulement à la structuration des lieux dans lesquels ce processus s'inscrit, mais aussi à leur connexion et à la création de nouveaux espaces. Or, si nous avons noté la présence de nombreux espaces de discussion (notamment informels) au sein de l'unité, ce qui facilite grandement le partage des difficultés rencontrées dans le travail, nous avons constaté, dans le même temps, des problèmes et des dysfonctionnements organisationnels. La seconde conversation est emblématique des problèmes qui se posent dans l'unité, à savoir : des prescriptions médicales qui ne sont pas toujours respectées par le personnel paramédical ; une mauvaise circulation de l'information (AS2 n'était par exemple pas au courant que la contention de Monsieur F. était une prescription médicale) ; des pratiques professionnelles hétérogènes ; des problèmes de communication et de coordination (notamment entre les différentes catégories de personnel). L'origine de ces problèmes est en partie due aux choix organisationnels qui ont été faits par l'encadrement. Ainsi, le fait que les transmissions orales se fassent par métier par

\footnotetext{
${ }^{5}$ II est fréquent que tel sujet abordé dans tel espace de discussion ressurgisse dans un autre lieu pour être à nouveau travaillé.
} 
exemple, et non de manière collective comme cela se pratique dans l'unité voisine, induit souvent des pertes d'informations et génère parfois des ajustements coûteux, voire des incompréhensions et des tensions (notamment entre les aides-soignants et les infirmiers). Notre enquête révèle également une très grande variabilité dans les pratiques de transmissions entre les aides-soignants ${ }^{6}$. Variabilité au niveau de la durée des transmissions tout d'abord, celles-ci pouvant aller de quelques minutes à peine à une vingtaine de minutes. Variabilité au niveau de l'ambiance sonore dans laquelle se déroulent les transmissions ensuite, l'environnement pouvant être tantôt propice à un échange de qualité, tantôt marqué par une certaine cacophonie. Variabilité au niveau de l'occupation de l'espace par les soignants enfin, les transmissions pouvant parfois se dérouler dans de très bonnes conditions (lorsque les aides-soignants sont assis l'un en face de l'autre ou l'un à côté de l'autre, en posture d'écoute et en miroir), parfois dans de très mauvaises conditions (distance interpersonnelle trop importante entre eux, informations transmises à la volée en station debout par un aide-soignant...). De ce fait, la quantité comme la qualité des informations transmises sont très variables ${ }^{7}$.

Pour pallier ces dysfonctionnements, nous avons émis un certain nombre de propositions qui visent à outiller la discussion sur le travail. Tout d'abord, dans la mesure où aucun espace de discussion n'a été prévu au niveau de l'unité pour traiter spécifiquement les incidents et dysfonctionnements organisationnels - même si, face à un problème donné, les acteurs peuvent toujours se réunir de manière ad hoc... -, nous suggérons d'introduire des retours d'expérience. Ouvrir ce type d'espace nous semble pertinent dans deux cas de figure :

(1) en cas d'événements indésirables, c'est-à-dire d'incidents mettant en jeu la sécurité des patients et/ou des soignants (comme le cas de la contention oubliée de Monsieur M. évoqué dans la conversation $\left.n^{\circ} 2\right)$;

(2) en cas de situations particulièrement complexes à gérer et à très forte charge émotionnelle (comme dans le cas de Madame $D$. évoqué dans la conversation $\left.n^{\circ} 1\right)^{8}$. Ensuite, nous suggérons de mettre en place des transmissions collectives impliquant aides-soignants et infirmiers, ce qui permettrait aux aides-soignants de mieux comprendre les contraintes des infirmiers et inversement, mais aussi de réduire la variabilité observée dans les pratiques de transmissions orales entre les aidessoignants. Nous proposons également d'introduire des «points de vigilance » dans les pratiques langagières orales et écrites, qui seraient énoncés de manière claire par le médecin lors du staff hebdomadaire (ce qui permettrait par exemple à l'ensemble du personnel paramédical d'être mieux informé au sujet des contentions). Ces « points de vigilance » seraient repris, non seulement lors des staffs suivants (de manière à réaliser un suivi dans le temps), mais viendraient également irriguer et structurer les transmissions orales, ce qui permettrait de coupler de manière plus étroite ces deux types d'espaces de discussion. Enfin, nous proposons de déployer un dispositif (quotidien) de type briefings/débriefings qui serait animé par les infirmiers, ceci afin, d'une part, de rappeler les « points de vigilance » et de déterminer la répartition des rôles entre infirmiers et aides-soignants pour la journée

\footnotetext{
${ }^{6}$ Une variabilité que nous n'avons pas observée dans le cas des transmissions entre infirmiers.

${ }^{7}$ La conversation $n^{\circ} 1$ donne à voir des transmissions orales qui donnent lieu à un échange de grande qualité. Mais il n'en est pas toujours ainsi.

${ }^{8}$ D'autant plus que la prise en charge de cette patiente induit de la souffrance (chez la patiente comme chez les soignants).
} 
à venir ${ }^{9}$ (lors du briefing), et, d'autre part, de permettre aux membres de l'équipe de s'exprimer sur la journée écoulée (lors du débriefing). Par ailleurs, nous avons mentionné, dans notre rapport, qu'il était important de maintenir la bonne ambiance de travail dans l'unité et de préserver la vitalité des espaces informels de discussion sur le travail.

\section{Discussion}

Nos recommandations ont été bien accueillies par le comité de suivi de la recherche qui a donné son accord pour que notre rapport soit transmis au pôle gériatrie de l'hôpital, moyennant quelques modifications mineures ${ }^{10}$. Elles devraient ensuite être discutées par l'ensemble des soignants de l'unité qui pourront, à leur tour, émettre des propositions de transformation de l'organisation du travail et/ou réfléchir aux modalités concrètes de mise en œuvre de nos recommandations. La démarche d'ensemble est donc très participative et a pour finalité ultime d'impulser une dynamique d'apprentissage collectif. Cette recherche-action est, en définitive, un pari sur l'intelligence collective.

Cette recherche apporte également des réponses aux trois questions posées dans l'introduction de cet article. Nous proposons tout d'abord une définition des espaces de discussion plus large que celle de Detchessahar (2011b, 2013) puisqu'elle inclut les espaces informels de délibération sur le travail. Ce faisant, elle tient compte des acquis de la psychodynamique du travail. Ce point nous semble d'autant plus capital que nous avons constaté que ces espaces constituaient des lieux dans lesquels souffle parfois un « vent de folie » comme en témoignent certaines de nos observations - nous y avons vu des soignants chanter, mimer certains patients, tourner en dérision certaines situations dramatiques, se moquer des collègues ou des chefs, pester contre la lourdeur de l'institution..., le tout dans une ambiance quasiment carnavalesque -, un « vent de folie » qui a toute sa place dans un univers où les soignants sont confrontés quotidiennement à la maladie et à la mort. Ces lieux sont donc bel et bien des espaces de régulation des tensions et des contradictions qui naissent de l'activité, d'autant plus que les soignants peuvent s'en emparer pour entamer des retours réflexifs sur l'activité (comme le montre la conversation $n^{\circ} 2$ ). A noter que ces espaces n'excluent pas, par principe, la présence de la hiérarchie, pour peu qu'elle respecte les pratiques de convivialité qui habitent ces lieux ${ }^{11}$. II convient donc de les intégrer pleinement à la réflexion, de les préserver et de maintenir leur vitalité, comme le recommande explicitement la psychodynamique du travail (Dejours, 1992, 2013).

Préserver les espaces informels de discussion sur le travail s'avère indispensable, mais n'est pas toujours suffisant si on veut résoudre certains dysfonctionnements organisationnels, comme par exemple les problèmes de circulation de l'information,

\footnotetext{
${ }^{9}$ La fiche de poste de l'aide-soignant mentionne qu'il « assure, en collaboration avec l'infirmier(e) et sous sa responsabilité, des soins de prévention, de maintien, de relation et d'éducation à la santé pour préserver et/ou restaurer la continuité de la vie, le bien-être et l'autonomie de la personne âgée. 》

${ }_{10}$ À la demande du comité, nous avons en effet supprimé certains extraits d'entretiens, ajouté quelques éléments de contexte au sujet de l'accompagnement des personnes en fin de vie et mieux mis en relief certains problèmes de gestion des ressources humaines. Quant à nos recommandations, le comité a souhaité les conserver telles quelles.

${ }^{11}$ Nous avons d'ailleurs constaté, sur notre terrain, que la cadre de santé, ainsi qu'un médecin, fréquentaient occasionnellement la salle de pause pour partager le café avec les aides-soignants et les infirmiers et évoquer les problèmes qui se posent dans le travail.
} 
de communication et de coordination entre les différentes catégories de personnel (des problèmes qui ne sont d'ailleurs en rien spécifiques à notre terrain). Proposer de structurer certains espaces, de les équiper et de les connecter davantage entre eux peut alors s'avérer nécessaire, comme nous proposons de le faire pour les staffs (en introduisant des " points de vigilance ») et pour les transmissions orales (en basculant sur des transmissions collectives). Nous faisons l'hypothèse qu'une telle action est de nature à fluidifier la circulation de l'information, à mobiliser davantage les acteurs autour d'objectifs communs, et à homogénéiser les pratiques professionnelles.

Il peut même être nécessaire, dans certains cas, de suggérer la mise en place de nouveaux espaces de discussion (à l'instar des retours d'expérience) afin de prendre en charge des problèmes qui sont complexes et que les acteurs peinent à résoudre selon les canaux habituels, soit parce qu'ils n'arrivent pas à partager leurs informations et leurs connaissances, à se coordonner et à apprendre des situations (comme dans le cas de la contention oubliée de Monsieur M. évoquée dans la conversation $\mathrm{n}^{\circ} 2$ ), soit parce que les situations rencontrées mettent à rude épreuve les travailleurs sur le plan émotionnel et génèrent de la souffrance au travail (comme dans le cas de la prise en charge de Mme D. évoquée dans la conversation $n^{\circ} 1$ ). Mais cela suppose d'être attentif au vécu émotionnel des travailleurs et à la manière dont ils mettent en mots leur expérience du travail et les affects qui les traversent. C'est d'autant plus important quand les situations de travail font naître des émotions et/ou des affects négatifs (angoisse, peur, dégoût, colère, etc.), comme c'est le cas dans l'univers hospitalier (Molinier, 2008, 2013). Nous rejoignons donc pleinement Briest-Breda et al. (2017) qui proposent de renouveler l'ingénierie des espaces de discussion en intégrant cette dimension affective et émotionnelle.

Déployer une ingénierie de la discussion sur le travail suppose de s'armer d'une méthode. Certaines actions que nous avons entreprises sont directement inspirées des recommandations de Detchessahar (2011b, 2013) en matière d'ingénierie des espaces de discussion, comme le fait de recenser ces espaces et de réfléchir à leur structuration. Cependant, notre méthode s'en différencie sur plusieurs points. En effet, tandis que Detchessahar et al. (2015) proposent d'institutionnaliser des espaces de discussion de niveau stratégique (donc de partir " d'en haut ») avant de se tourner vers le niveau opérationnel, notre démarche, elle, part « d'en bas » et cherche avant tout à répondre à une demande locale ${ }^{12}$. De ce point de vue, elle est beaucoup plus proche de l'enquête en psychodynamique du travail qui repose sur la mise en place d'un « collectif d'enquête » (Begue et Dejours, 2009). Par ailleurs, tandis que pour Detchessahar et al. (2015), l'intervention vise avant tout à mettre en place de nouveaux espaces de discussion, et non à analyser le travail, notre méthode, elle, est fondée sur les suivis d'activités (Guerin et al., 1997), et donc sur l'analyse du travail ${ }^{13}$. Cette méthode est certes lourde et chronophage, mais elle permet de réaliser un inventaire très précis des espaces de discussion (formels et informels) existant dans l'entité étudiée et des dynamiques communicationnelles qui s'y déploient. Elle permet aussi de repérer les dysfonctionnements existant au niveau de la communication et offre une prise pour (re)penser les espaces de discussion et leur agencement.

\footnotetext{
${ }^{12}$ La démarche déployée est en fait contingente à la demande.

${ }^{13}$ À l'instar de la psychodynamique du travail.
} 


\section{Bibliographie}

ABORD DE CHATILLON E. \& DESMARAIS C. (2016), « Espaces de discussion, management et santé au travail », Document de la chaire management et santé au travail. http://www.chaires-iae-grenoble.fr/mansat/documents.php

ABORD DE CHATILLON E. \& DESMARAIS C. (2017), « Espaces de discussion, management et épuisement professionnel », in Revue @GRH, n²3, pp. 13-36.

BEGUE F. \& DEJOURS C. (2009), Suicide et travail : que faire ?, PUF.

BELLINI S., DREVETON B., GRIMAND A. \& OIRY E. (2018), "Les espaces de discussion : un vecteur de régulation des paradoxes de la nouvelle gestion publique ? ", in Revue de gestion des ressources humaines, $\mathrm{n}^{\circ} 107$, pp. 3-22.

BORZEIX A. (1987), "Ce que parler peut faire », in Sociologie du travail, n², pp. 157-176.

BORZEIX A. (2001), “ Le travail et sa sociologie à l'épreuve du langage », in BORZEIX A. \& FRAENKEL B. (dir.), Langage et Travail : Communication, cognition, action, CNRS Editions.

BRIEST-BREDA C., RICHARD D. \& YANAT Z. (2017), « Design Thinking et Théorie U : vers une nouvelle ingénierie des espaces de discussion pour développer le mieux-être au travail ? », in Management \& Sciences Sociales, n²2, pp. 38-55.

CLOT Y. (2008), Travail et pouvoir d'agir, PUF.

CLOT Y. (2010), Le travail à cœur, Pour en finir avec les risques psychosociaux, La Découverte.

CONJARD P. (2015), Le management du travail : Une alternative pour améliorer bien-être et efficacité au travail, Editions Anact.

CONJARD P. \& JOURNOUD S. (2013), « Ouvrir des espaces de discussion pour manager le travail », in Management \& Avenir, n63, pp. 81-97.

DEJOURS C. (1992), "Pathologie de la communication, situation de travail et espace public : le cas du nucléaire », in Raisons pratiques, vol. 3, pp.177-201.

DEJOURS C. (1998), Souffrance en France : la banalisation de l'injustice sociale, Seuil.

DEJOURS C. (2013), Travail vivant - 2. Travail et émancipation, Editions Payot \& Rivages.

DETCHESSAHAR M. (dir.) (2009), Etude SORG : Santé, Organisation et Gestion des

Ressources humaines, Agence Nationale de la Recherche (Programme SEST Santé-

Environnement Santé-Travail 2006-2008).

DETCHESSAHAR M. \& GREVIN A. (2009), « Un organisme de santé... malade de "gestionnite" ", in Gérer et Comprendre, n 98, pp. 27-37.

DETCHESSAHAR M. (2011a), "Management et santé », in Revue française de gestion, n²14, pp. 65-68.

DETCHESSAHAR M. (2011b), “Santé au travail. Quand le management n'est pas le problème, mais la solution... ", in Revue française de gestion, n²14, pp. 89-105.

DETCHESSAHAR M. (2013), « Face aux risques psychosociaux, quelques éléments d'un management par la discussion », in Négociations, n¹9, pp. 57-80.

DETCHESSAHAR M., GENTIL S., GREVIN A. \& STIMEC A. (2015), « Quels modes d'intervention pour soutenir la discussion sur le travail dans les organisations ? Réflexions méthodologiques à partir de l'intervention dans une clinique », in @GRH, n¹6, pp. 63-89.

DETCHESSAHAR M., GENTIL S., GREVIN A. \& JOURNE B. (2017), « Entre cacophonie et silence organisationnel, concevoir le dialogue sur le travail. Le cas de projets de maintenance dans une industrie à risque ", in Gérer et comprendre, n 130, p. 33-45. 
DEWEY J. (1993 [1938]), Logique. La théorie de l'enquête, PUF.

FUSTIER P. (2012), " L'interstitiel et la fabrique de l'équipe », in Nouvelle revue de psychosociologie, $\mathrm{n}^{\circ} 14$, pp. 85-96.

GIUST-OLLIVIER A.C. \& OUALID F. (dir.) (2011), « Les groupes d'analyse des pratiques », Nouvelle Revue de Psychosociologie, $\mathrm{n}^{\circ} 11$.

GROSJEAN M. \& LACOSTE M. (1999), Communication et intelligence collective. Le travail à l'hôpital, PUF.

GUERIN F., LAVILLE A., DANIELLOU F., DURAFFOURG J. \& KERGUELEN A. (1997), Comprendre le travail pour le transformer. La pratique de l'ergonomie, Editions de l'ANACT. JOURNE B. (2007), «Théorie pragmatiste de l'enquête et construction du sens des situations », Le Libellio d'AEGIS, n4, pp. 3-9.

JOURNE B. \& RAULET-CROSET N. (2008), « Le concept de situation : contribution à l'analyse de l'activité managériale dans un contexte d'ambiguïté et l'incertitude », M@n@gement, n¹, pp.27-55.

MOLINIER P. (2008), Les enjeux psychiques du travail, Editions Payot.

MOLINIER P. (2013), Le travail du care, La Dispute.

RICHARD D. (2012), Management des risques psychosociaux : une perspective en termes de bien-être au travail et de valorisation des espaces de discussion, Thèse de doctorat, Université de Grenoble.

THEUREAU J. \& JEFFROY F. (1994), Ergonomie des situations informatisées, Toulouse, Octares. 